\title{
Quantum Continuous Gradient Models in the Study of Markets
}

\author{
Javier M. Huarca Ochoa \\ University of San Martín de Porres \\ Luis H. Ludeña Saldaña \\ University of San Martín de Porres
}

Business students graduate without knowing about the existence of quantum continuous gradient models (QCGM) to study financial markets. This paper introduces and discusses these models. The underlying function space Map (X,Y) of QCGM is a set of smooth maps called envelope-gradient functions (EGF) from $X$ to $Y$ with the standard compact-open topology. Herein, we take advantage of some natural properties of EGF to define a classical associative algebra on it and develop a mathematical QCGM. The development of QCGM involves principles of deformation quantization theory and definite integrals of EGF on uniform probability distributions. Applications in economics and further lines of research are suggested.

Keywords: gradient functions, differentiable Poisson manifolds, quantization deformation, associative algebras, generalized law of supply and demand

\section{INTRODUCTION}

Over two centuries ago, power tower functions (PTF) were studied by L. P. Euler (1783) and G. Eisenstein (1844), and many mathematicians have continued writing papers about PTF to this day, namely Le Lionnais (1983), Wells (1986, p. 35), Vardi (1991), Olver et al. (2010), and L. Moroni (2019). So, there is an abundant bibliography on these uncanny functions, which are known under such different names as "Infinite Exponentials" (Rippon, 1983), "Iterated Exponential Constants" (Finch, 2003), etc. In this paper, we purposely call them Envelope-Gradient Functions (EGF).

This paper arises from the attempt to study some natural geometric and algebraic properties of finite level smooth envelope gradient functions on a Poisson manifold and use these findings to construct quantum continuous gradient models (QCGM) and establish some of their principal features.

The general framework of the QCGM is a function space Map (X, Y) on a manifold M whose members constitute a set of continuous maps from $\mathrm{X}$ to $\mathrm{Y}$ with the standard compact-open topology. These members are precise: the EGFs. Here the purpose of the word 'envelope' is to point out the geometric framework underlying our discussion, the domain of the uniform distribution of probability (see Figure 1). These EGFs, whose shapes and behavior are similar to those of well-known elementary functions, also comply with the rules of differential and integral calculus such as the central concepts of limits, continuity, derivatives, integration, and power series. One of the first and most obvious properties of EGFs is that all members share a common fixed point. Namely, all EGFs have a single attractive fixed point $(1,1)$ at the north-eastern vertex of the envelope seated on the unit square $I \times I$, as depicted in Figure 1, where $I$ is the interval $[0,1]$. 
More generally, for our purposes, $I_{\alpha}=[0, \alpha]$, for $\alpha \in(0,1) \cup\{1,2,3, \ldots\}$. These sets will be, precisely, the fundamental domain of the uniform distributions and the target of each function space EGF on which most of the work and exploratory analysis of the QCGM is done.

The study procedure is as follows: Firstly, we define the EGF and review some of its relevant properties to define a classical associative commutative algebra of EGF on a differentiable Poisson manifold M. It will be the setting for most of our discussion, in which we compute some definite integrals of EGF on uniform probability distributions, and the setting where we deal with purely formal algebraic properties of deformation quantization of this classical algebra as a formal power series. In the first half of this procedure, we will show that the integration of lower-level EGFs, and probably also high-level ones, is strongly linked to several well-known special mathematical functions, such as Gamma, Beta, Whittaker M, Confluent Hypergeometric Function, and others. However, we don't intend to further explore these interesting relations in this paper. We will present these studies and findings elsewhere.

Secondly, after we highlight the meaning of "deformation quantization" we proceed to deform the previously defined classical associative commutative algebra of EGFs with the purpose of performing the same analysis we have done on this classical algebra. Thus, in the second half of this procedure description we point out a few concepts and some principles of quantum mechanics formalism.

Furthermore, throughout the integral-solving process, we illustrate the principle of finite discreteness in quantum mechanics and the principle of correspondence between classical and quantum fields with the existence of the classical limit. Thirdly, we combine both findings, two world results, to compare and conclude the QCGM features.

Finally, we present a concrete case for the previous steps, to explore and discuss - both from a classical and quantum point of view - some topics in market economics and finance in the context of some uniform probability distributions. Specifically, we explore the generalized law of supply and demand from this new point of view describing many distinct areas under (over) the shape of lower-level EGFs (e.g., we explore areas of consumer and producer surplus, surplus loss, the intensity of demand and supply, etc.) on several domains of $I \times I$.

This paper is organized as follows: Section 2 provides the required existing literature review and the postulates for the development of the QCGM. Section 3 shows the QCGM construction procedure over several domains geometrically resembling envelopes bounded and crossed by gradients (Figure 1). Next, to support this methodology, Section 4 presents a concrete application of the QCGM. Finally, Section 5 concludes with a summary of findings, as well as some recommendations and suggestions for further research.

\section{REQUIRED SUPPORTING LITERATURE}

In this section, before formally developing the quantum continuous gradient market models, a review of the literature providing the theoretical basis is presented. Specifically, we present some concepts and properties of envelope gradient functions, as well as a very brief review of quantization formalism and deformation quantization. Also, we briefly introduce the standard concept of the generalized law of supply and demand.

\section{Envelope Gradient Functions}

Envelope gradient functions (EGF), which have historically been given varying denominations, have been studied for more than two centuries, starting with L. Euler (1783) and G. Eisenstein (1844). Since

then, many other mathematicians have continued researching up to the present day and, nowadays, EGFs are being applied in several computer software languages and can be computed analytically (Knuth, D. E., 1976).

\section{Definition $A$}

Let $G_{k}(x)$ denote the real-valued continuous gradient function of level $k$, where $k$ is a finite natural number. Then, $G_{k}(x)$ is called an envelope gradient function defined by: 
$G_{k}(x)=\underbrace{x^{x^{x^{x^{x}}}}}_{k}$ for $x \geq 0$.

Here, $G_{k}(x)$ is evaluated from the top-down a finite number of times and each subsequent level $x$ is adjoined to the bottom of the gradient. EGFs may be unfamiliar to the business community. Therefore, a few examples of shapes, for $k=1,2,3,4$, and properties are provided to clarify the definition and notation of these functions. Also, as most computer software does, hereinafter we assume that $0^{0}=1$.

$G_{1}(x)=x, \mathrm{G}_{2}(x)=x^{x}, \mathrm{G}_{3}(x)=x^{x^{x}}, \mathrm{G}_{4}(x)=x^{x^{x^{x}}}$, for each $x \geq 0$, show the first, second, third, and fourth-level EGF, respectively. Their shapes are shown in Figure 1.

From Definition A and Figure 1, it would seem that for $x>1 G_{k}(x)$ must diverge. But, amazingly, this is not so (Galidakis, I. N. 2006). For our purposes, $G_{k}(x)$ has a finite number of levels and real values in the uniform distribution over $I_{x}$. Also, it is well defined on the domain $0 \leq x \leq e^{\frac{1}{e}}$ and any closed and bounded interval $I_{x}$ for $x$, as above.

\section{FIGURE 1}

\section{GEOMETRIC FRAMEWORK SHOWING SHAPES OF SOME EGF FOR $K=1,2,3,4$,}

$\operatorname{In}[12]:=\operatorname{Plot}\left[\left\{x, x^{\wedge} x, x^{\wedge}\left(x^{\wedge} x\right), x^{\wedge}\left(x^{\wedge}\left(x^{\wedge} x\right)\right)\right\},\{x, 0,1.5\}\right.$, AxesLabel $\rightarrow$

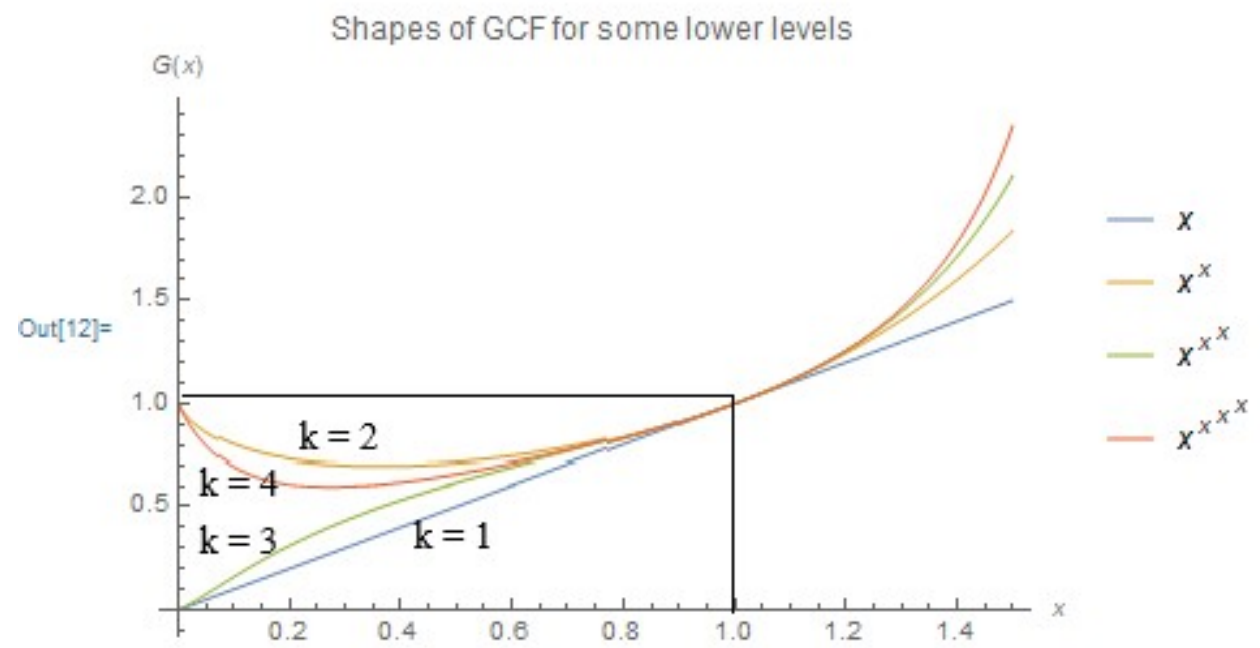

We plot a few shapes of lower-level continuous gradient functions $G_{k}(x)$. For illustration purposes, we have partially enclosed their targets by a unit square $I_{1} \times I_{1}$, the main 'envelope' of the discussion.

If we keep adjoining more levels at the bottom of the EGF, it produces an infinite sequence of successive approximations to (1). So, this sequence converges on the domain established already above. Enough papers are proving these facts and iterative solutions for a given $x$ (Lynch, 2013). EGF's fundamental property, for our purpose, is that the terms of the subsequence $\left\{G_{2 k-1}(x)\right\}$ are strictly increasing monotonic functions, but the terms of the subsequence $\left\{G_{2 k}(x)\right\}$ are not (see Lemma 1). Therefore, gradient functions should be understood as a sequence of functions whose odd members increase monotonically on the entire domains and whose even members do not. Additionally, even members share a local extreme value when $x=e^{-1}$.

\section{Quantum Mechanics Formalism}

Here, we briefly present a general conceptualization of the formalism of quantum mechanics (QM) and then we focus on the quantization deformation method initiated by Bayen F. et al. (1978). The QM 
formalism establishes the meaning of quantization and it tells us that there are many quantization algorithms complying with the correspondence principle (Berezin F.A., 1975), all of which describe the evolution of a system. An intuitive method to quantizing a classical mechanic's dynamic system should be the following:

Let $(M, \tau)$ be a classical mechanics model where $M$ is a differentiable manifold and $\tau$ is a skewsymmetric tensor field on this manifold. Let $A(M)$ be a set of differentiable functions $f, g, \ldots$, on $M . A(M)$ is a commutative and associative algebra for the standard addition and multiplication and it is a Lie algebra concerning the Poisson bracket $\{f, g\}=\tau^{i j} \frac{\partial f}{\partial x^{i}} \frac{\partial g}{\partial x^{j}}$ where de Jacobi identity is valid.

With these foundations, we borrow some ideas from F. Berezin's paper (1975) and give a general mathematical definition of quantization, from a deformation quantization viewpoint.

\section{Definition $B$}

The associative noncommutative algebra $\mathfrak{A}$ with involution is identified as the quantization of the classical mechanics $(M, \tau)$ if it has the following properties: (1) there is a family $A_{\hbar}$ of associative algebras, of differentiable functions $f(x)$ for $x$ in $M$, such that the index $\hbar$ is the formal variable and runs on the positive side of the real axis and $\mathfrak{A}$ consists of functions $f(h, x)$ taking values in $A_{\hbar}$, for fixed $\hbar$. The involution and multiplication in $\mathfrak{A}$ are connected with the involution and multiplication of $A_{\hbar}$ in the usual way $\left(f^{\odot}\right)(\hbar)=(f(\hbar))^{\bullet}$, where $\odot, \bullet$ are the involutions in $\mathfrak{A}$ and $A_{\hbar}$ respectively, $\left(f_{1} \otimes f_{2}\right)(\hbar)=$ $f_{1}(\hbar) \times f_{2}(\hbar)$, and where $\Theta, \times$ are the multiplications in $\mathfrak{U}$ and $A_{\hbar}$, respectively. (2) There is a homomorphism $\phi: \mathfrak{U} \rightarrow A(M)$ of the differentiable functions on $M$, with the standard + and $\times$, given by $\phi(f)=\lim _{\hbar \rightarrow 0} f(\hbar, x) k=1$ and meeting: $(i)$ for any two points $x_{1}, x_{2}$ in $M$, there is a function $f(x)$ in $\phi(\mathfrak{U})$ such that $f\left(x_{1}\right) \neq f\left(x_{2}\right)$. (ii) $\phi\left(\frac{1}{h}(f * g-g * f)\right)=i\left[\phi\left(f_{1}\right), \phi\left(f_{2}\right)\right]$, where $*$ denotes the multiplication in $\mathfrak{A},[.,$.$] is the Poisson bracket in A(M)$, and $i=\sqrt{-1}$. (3). The unit of algebra $A_{\hbar}$ is the function $f(\hbar, x) \equiv 1$.

It is understood that this conceptualization of algebraic deformation quantization also involves and supports the four main old principles of QM that need to be recalled. Based on the legacy of both Heisenberg's initial approach to quantum mechanics in 1925 and Bohr's (1913) initial interpretation of the theory, offered in 1927, we have been provided with the following principles (A, Plotnitsky, 2016): (1) The principle of quantum discreteness (QD) states that all quantum phenomena, defined as what is observed through measuring instruments, are individual and discrete, which is not the same as the (Democretian) atomic discreteness of quantum objects themselves. This QD principle originated in Bohr's 1913 theory of the hydrogen atom as based on "quantum postulates" of the discrete behavior ("quantum jumps") of electrons in atoms; (2) the principle of quantum probabilistic and statistical (QPS) nature of quantum predictions states that all quantum predictions are of this nature, even in the case of elementary individual quantum processes and events, such as those associated with elementary particles; (3) the correspondence principle (CP), which was initially used by Bohr and others, stated that the predictions of quantum theory must coincide with those of classical mechanics at the classical limit when Planck's constanth $\rightarrow 0$, but was given by Heisenberg a mathematical form, which required both the equation and variables used to be converted into those of classical mechanics at the classical limit; and (4) the principle of complementarity (PC), added to the first three principles. The complementarity principle says that parts never add up to a whole in the way they do in classical physics or relativity, given that at any moment only one of these parts could be ascertained, and hence is the only "whole" at this moment. In the formalism of quantum mechanics, the non-commutativity of the multiplication of the corresponding variables, as the position and momentum operators, could be seen as the mathematical expression of the complementarity principle. We intend to show the presence of some of these principles while expanding on and applying the QCG models to markets. However, instead of building a Hilbert space from a Poisson manifold and associating radical changes to original observables, we are only concerned with the algebras. After all, both systems can be studied in terms of their algebras. [3] It is proposed to deform the commutative standard product of algebra $C^{\infty}(M)$ into a non-commutative, associative product. To clarify the context in which we will develop the models, we define Poisson manifolds and formal deformation of associative algebras. 


\section{Definition $C$}

A Poisson manifold is a manifold $M$ whose algebra $A=C^{\infty}(M)$ of smooth functions on it is Poisson algebra with pointwise multiplication as a commutative product. Poisson algebra is a vector space $V$ equipped with a commutative, associative product and a Lie bracket. In other words, for each $f, g, h$ in $C^{\infty}(M)$ the commutative product $(f, g) \rightarrow f g$ makes $V$ into a commutative algebra and the Poisson bracket $(f, g) \rightarrow\{f, g\}$ makes $V$ into a Lie algebra that complies with the Jacobi Identity and Leibniz rule of derivation (see Endnotes).

\section{Definition $D$}

Fix a field $\mathrm{K}$ of characteristic zero. Let $A=C^{\infty}(M)$ be an associative algebra, as above. A formal deformation of $A$ is an associative $K \llbracket \hbar \rrbracket$-algebra structure on $A \llbracket \hbar \rrbracket$ such that $A \simeq A \llbracket \hbar \rrbracket / \hbar$ as algebras. Establish that for any $f, g \in A, f * g=f g+B_{1}(f, g) \hbar+B_{2}(f, g) \hbar^{2}+\ldots+B_{n}(f, g) \hbar^{n}+\ldots$, where: (1) the star product $*$ is a deformed product in $A$ extended to the algebra $A \llbracket \hbar \rrbracket$ by the $K \llbracket \hbar \rrbracket$-linearity, * $: A \llbracket \mathrm{\hbar} \rrbracket \times A \llbracket \hbar \rrbracket \rightarrow A \llbracket \mathrm{h} \rrbracket$ which takes on the particular value on $A$ when the parameter of deformation $\rightarrow 0$. In this case, $f * g=f g=B_{0}(f, g)$. (2) $B_{n}: A \otimes A \rightarrow A$ are bidifferential operators, i.e., bilinear maps which are differential operators for each argument.

\section{DEVELOPMENT OF QCG MODELS}

Returning to our goal, we organize this section as follows: Firstly, we construct Type I QCG Models, secondly, Type II QCG Models, and thirdly, Type III QCG Models. To initiate these developments, we fix a Poisson manifold $M=\mathbb{R}_{++}^{2} \subset \mathbb{R}^{2} \subset \mathbb{R}^{d}$, where $\mathbb{R}_{++}^{2}=\mathbb{R}_{+} \times \mathbb{R}_{+}$is the first quadrant subset of the plane $\mathbb{R}^{2}$ on which the observables (classical and quantum), depending on the system's state, are the set of smooth gradient functions $C^{\infty}(M)=\left\{G_{k}(x): x \geq 0, k=1,2,3, \ldots\right\}$. This Poisson manifold is our work's fundamental framework. We use these models to study and explore a system's evolution with these models in terms of both classical and quantum mechanics.

The general procedure consists of starting with Definition A and constructing a commutative associative classical algebra. Then, proceed to deform this algebra to obtain noncommutative associative quantum algebras. Thereafter, we deal with purely formal algebraic structure properties of EGF. So, we begin with the classical one.

\section{Type I QCG Models}

In this case, the procedure is clear. It only requires the Poisson manifold $\mathbb{R}_{++}^{2}$ and the smooth gradient functions $G_{k}(x)$ of level $k=1,2,3, \ldots$

\section{Lemma 1}

If $G_{2 k}(x)$ and $G_{2 k-1}(x)$ are integrable gradient functions of even (odd) level, respectively, on the closed interval $[0,1]$ and $0 \leq G_{2 k-1}(x) \leq G_{2 k}(x)$, for each level $k$, then

$$
0 \leq \int_{0}^{1} G_{2 k-1}(x) d x \leq \int_{0}^{1} G_{2 k}(x) d x
$$

This Lemma ensures the preservation of inequality under the integration - notice that its proof can be read in any calculus book (e.g., Larson R. et al., 2006, pg. 278). Here, we have adapted it to these particular gradient functions to support the following theorem. 


\section{Theorem $H$}

Let $\left\{G_{k}(x)\right\}$ be a sequence of differentiable gradient functions of nonnegative terms on the closed interval $[0,1]$. Let $\left\{G_{2 k}(x)\right\}$ and $\left\{G_{2 k-1}(x)\right\}$ be two subsequences of $\left\{G_{k}(x)\right\}$ such that $G_{2 k-1}(x) \leq$ $G_{2 k}(x)$ for each $k=1,2,3, \ldots$, then, $\lim _{k \rightarrow \infty}\left|\int_{0}^{1}\left(G_{2 k}(x)-G_{2 k-1}(x)\right)\right| \rightarrow 0$.

We should call this theorem 'Zipper Theorem'. In Figure 1, we see that all terms of this sequence meet at their fixed point $(1,1)$. Hence, their distance at this point is zero (the beginning of the open zipper). However, as $x$ gets closer and closer to zero from the right side, the distance between $G_{2 k}(x)$ and $G_{2 k-1}(x)$ becomes larger and larger, for each $k$. Fortunately, the distance between the boundaries of this gap is finite (such as a real zipper). In other words, the area bounded by an open zipper and the vertical $G_{k}(x)$ - axis gets smaller and smaller as $k$ takes on higher values. All these dynamic sequences of functions should be a setting for studying classical and quantum dynamic economics, i.e., financial markets.

Proof

Firstly, we show that the integral inside the absolute value on the closed interval $[0,1]$ exists. However, this part of the proof is supported by Lemma 1. Secondly, we consider partitioning $0=x_{0}<x_{1}<x_{2}<$ $\ldots<x_{i-1}<x_{i}<\ldots<x_{n}=1$ of $[0,1]$ into $n$ subintervals, each of width $\Delta x=\frac{1}{n}$. Let $c_{i}$ be the center of the $i$ th subinterval. We can approximate the distance (height), $h_{i}=G_{2 k}\left(c_{i}\right)-G_{2 k-1}\left(c_{i}\right)$ between the curves $G_{2 k-1}(x)$ and $G_{2 k}(x)$, involved in the $i$ th subinterval. So, the area of this sub-rectangle is $h_{i} \Delta x$. Hence, through the Riemann sum, we can approximate the total area bounded below by the curve $G_{2 k-1}(x)$, above by the curve $G_{2 k}(x)$, and the vertical axis, for each $k$ (see Figure 1). So, when the levels $k=1,2,3, \ldots$ of the functions $G_{k}(x)$ increase, the target of $\left\{G_{2 k}\left(c_{i}\right)\right\}$ decreases and the target of $\left\{G_{2 k-1}\left(c_{i}\right)\right\}$ increases on the interval $[0,1]$, by Lemma 1 . So, they will meet somewhere within the limit when $k \rightarrow \infty$. Since $c_{i}$ is arbitrary for $i=1,2,3, \ldots, n$, it follows that:

$$
\lim _{k \rightarrow \infty}\left|G_{2 k}\left(c_{i}\right)-G_{2 k-1}\left(c_{i}\right)\right|=0 \text { or } \lim _{k \rightarrow \infty}\left|\int_{0}^{1}\left(G_{2 k}(x)-G_{2 k-1}(x)\right)\right| \rightarrow 0
$$

To support this general concept, we now compute some areas between (below, above) the curves of $G_{k}(x)$. These computations of integrals will show us the amazing relation between EGF $G_{k}(x)$ and several special mathematical functions and the passage from continuous to discrete, the QD principle. Thus, we transform some of the EGFs of the space Map $(X, Y)$, applying simple definite integral operators on the interval $(0, b)$, as follows:

$\int_{0}^{b} G_{k}(x) d x$, when $k=1,2,3,4 \ldots$ and $0<x \leq b<\infty$

For our purposes, we make use of the obvious case $G_{1}(x)=x$, which is the well-known elementary Identity Function who's integral and applications are well known to all elementary calculus students. The interesting and intriguing results of integrals of gradient functions begin when $k=2$. In fact, starting with the integral in (2), we write its integrand as uniformly convergent power series and integrate term by term:

$$
\int G_{2}(x) d x=\int x^{x} d x=\int e^{x \ln x} d x=\int \sum_{p=0}^{\infty} \frac{(x \ln x)^{p}}{p !} d x=\int d x+\int \frac{x \ln x}{1 !} d x+\int \frac{x^{2} \ln ^{2} x}{2 !} d x+\ldots
$$

Next, we evaluate each integral and associate their results by patterns:

$$
=\left[x-\frac{x^{2}}{2^{2}}+\frac{x^{3}}{3^{3}}-\frac{x^{4}}{4^{4}}+\ldots\right]+\frac{\ln x}{1 !}\left[\frac{x^{2}}{2}-\frac{x^{3}}{3^{2}}+\frac{x^{4}}{4^{3}}-\ldots\right]+\frac{\ln ^{2} x}{2 !}\left[\frac{x^{3}}{3}-\frac{x^{4}}{4^{2}}+\ldots\right]+\ldots
$$




$$
=\sum_{p=1}^{\infty} \frac{(-1)^{p-1} x^{p}}{p^{p}}+\frac{\ln x}{1 !} \sum_{p=2}^{\infty} \frac{(-1)^{p-2} x^{p}}{p^{p-1}}+\frac{\ln ^{2} x}{2 !} \sum_{p=3}^{\infty} \frac{(-1)^{p-3} x^{p}}{p^{p-2}}+\ldots=\sum_{m=0}^{\infty} \frac{\ln ^{m} x}{m !} \sum_{p=m+1}^{\infty} \frac{(-1)^{p-m-1} x^{p}}{p^{p-m}}
$$

Now, we shift the right-hand series to the origin, agreeing that $n=p-m-1$, and we obtain

$$
\int x^{x} d x=\sum_{m=0}^{\infty} \frac{\ln ^{m}(x)}{m !} \sum_{n=0}^{\infty} \frac{(-1)^{n} x^{m+n+1}}{(m+n+1)^{n+1}}=H(m, n ; x)
$$

It is a double series of functions $H(m, n ; x)$ that gives rise to two infinite families of function series. To be more precise, the infinite members of the $m$-family of series arise varying $m$ from 0 to $\infty$ for each fixed $n=0, n=1, n=2, \ldots$ and the infinite members of the $n$-family arise varying $n$ from 0 to $\infty$ for each fixed $m=0, m=1, m=2, \ldots$. It is easy to show that the double series in (3) is decreasing and by the ratio test $\lim a_{m, n}=0$ as $m, n$ tends to $\infty$. For a fixed $x=\exp (-1)$, critical value of $\mathrm{G}_{2}$, some members from each family are plotted in Figure 2.

FIGURE 2

\section{GEOMETRIC VISUALIZATION OF SOME MEMBERS OF THE $(M, N)$ SERIES}

$$
\text { Family of } \mathrm{H}[\mathrm{m}, \mathrm{n}, \mathrm{x}] \text { Functions }
$$

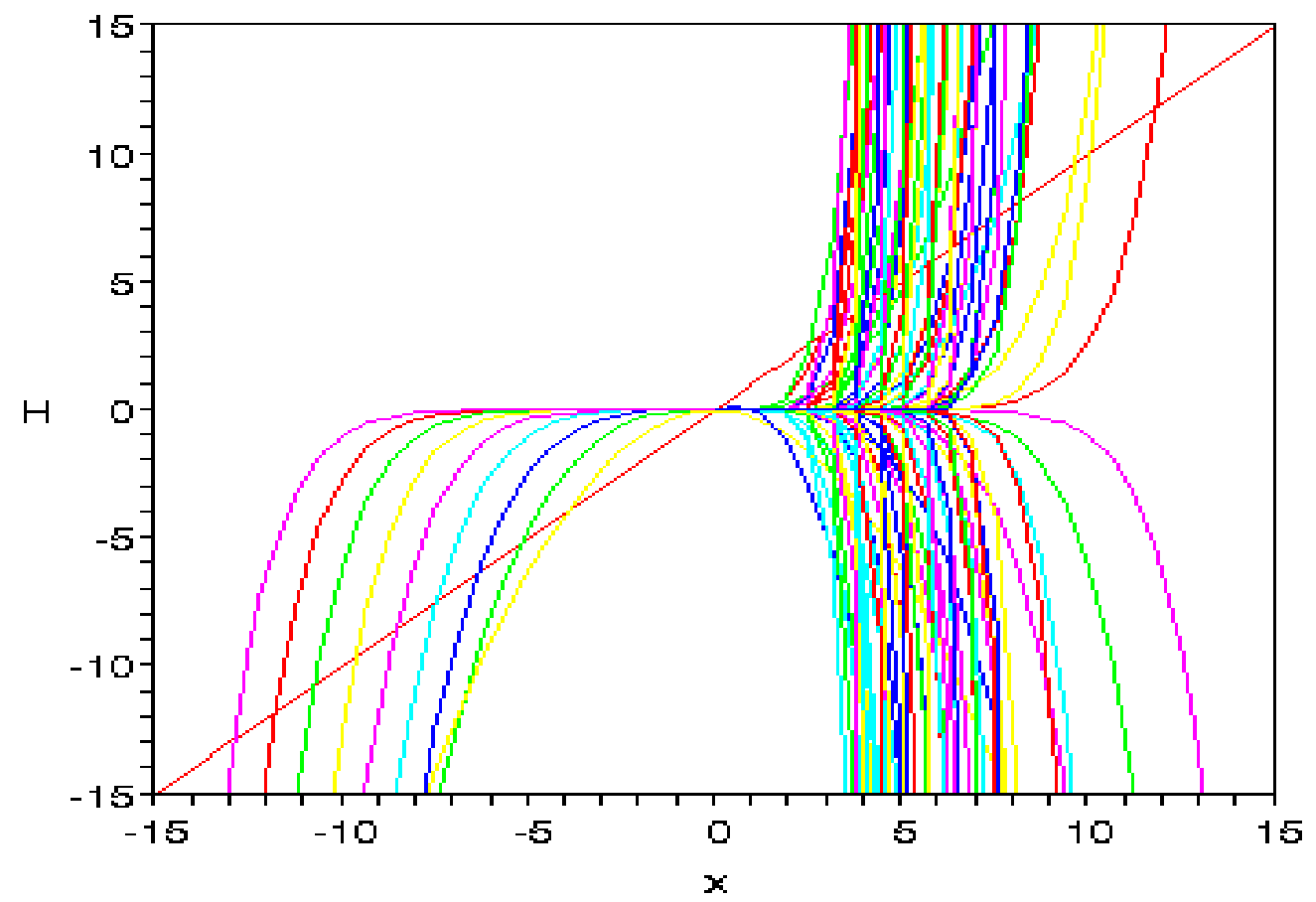

Plot of some series members of $H(m, n ; x)$, each for a fixed pair $(m, n)$, where $m, n=0,1 \ldots, 10$. We can see shapes of polynomial functions and shapes of the product of polynomial and logarithmic functions providing an amazing picture of a "symmetrical fall". The diagonal line is the identity function resulting when $m=n=0$.

From (3), many interesting function series arise. Namely, form $=0$ or $n=0$, respectively,

$$
H(0, n ; x)=\sum_{n=1}^{\infty} \frac{(-1)^{n-1} x^{n}}{n^{n}} \quad \text { and } \quad H(m, 0 ; x)=\sum_{m=0}^{\infty} \frac{x^{m+1} l^{m} x}{(m+1) !}
$$


Notice that $H(0, n ; x)$ is precisely a generalization of "Sophomore's dream", so that:

$\sum_{n=1}^{\infty} \frac{(-1)^{n-1} x^{n}}{n^{n}} \mid \quad{ }_{0}^{1}=H(0, n ; 1)-H(0, n ; 0)=1-\frac{1}{2^{2}}+\ldots-\frac{1}{10^{10}}=\frac{40953336089635928267832533257}{52274369621610823680000000000}$

A direct computation of the left-hand of (3) with Maple immediately verifies our result and, surprisingly, we can see that this integral relates to the integral-valued Gamma Function $\Gamma(n)$ and other special functions. As a special case, we have:

$\int_{0}^{1} x^{x} d x=\sum_{n=0}^{\infty} \frac{(-1)^{n}(n+1)^{-n-1} \Gamma(n+1)}{n !}=\frac{32785}{41848} \approx 0.783430510418658 \ldots$

So, the result in (5) agrees with the result in (6). Another interesting series arises when we set $m=n$ in $H(m, n ; x)$, with the 'Diagonal Series' of the lattice $m \times n$ for $m, n=0,1,2,3, \ldots$

$H(n, n ; x)=H_{D}(n, x)=\sum_{n=0}^{\infty} \frac{(-1)^{n} x^{2 n+1} l^{n} x}{n !(2 n+1)^{n+1}}$ for $x>0$

Notice that this series and the one on the right hand of (4) vanish for $x=1$, the fixed point of all gradient functions. Consistently, all series in (3) vanish when $x=1$, except the series on the left hand of (4), which is the only polynomial series in these infinite families. This explains why it is only this series which contributes rapidly to the approximation of the integral (6).

Notice also that in (2), when $k=3$ and following the same strategy, we can evaluate the integral of third-level gradient function $G_{3}(x)=x^{x^{x}}$ for $x \geq 0$ as follows:

$$
\begin{aligned}
& \int G_{3}(x) d x=\int x^{x^{x}} d x=\int e^{\left(x^{x} \ln x\right)} d x=\int \sum_{q=0}^{\infty} \frac{\left(x^{x} \ln x\right)^{q}}{q !} d x=\int \sum_{q=0}^{\infty} \frac{x^{q x} \ln ^{q} x}{q !} d x \\
& =\int \sum_{q=0}^{\infty} \frac{e^{(q x \ln x)} \ln ^{q} x}{q !} d x=\int \sum_{q=0}^{\infty} \sum_{p=0}^{\infty} \frac{(q x \ln x)^{p} \ln ^{q} x}{p ! \mathrm{q} !} d x=\int \sum_{q=0}^{\infty} \sum_{p=0}^{\infty} \frac{q^{p} x^{p} \ln ^{p+q} x}{p ! \mathrm{q} !} d x
\end{aligned}
$$

Next, assuming conditions of convergence, we interchange summation and integration and proceed to evaluate:

$$
\int x^{x^{x}} d x=\sum_{q=0}^{\infty} \sum_{p=0}^{\infty} \frac{q^{p}}{p ! q !} \int x^{p} \ln ^{p+q} x d x
$$

Once again, we let $b=1$ in (2) and evaluate the definite integral, via Maple, obtaining:

$$
\int_{0}^{1} x^{x^{x}} d x=\sum_{q=0}^{\infty} \sum_{p=0}^{\infty} \frac{q^{p}}{p ! q !}(-1)^{p+q}(1+p)^{-(p+q+1)} \Gamma(p+q+1) .
$$

Notice that if $q=0$ and $p>0$ this double series vanishes to zero. Letting $s=p+1$, we rewrite (8) and observe that this integral relates to the Gamma Function and also to the special widely-known Beta function $B$. Thus, we have:

$$
\int_{0}^{1} x^{x^{x}} d x=\sum_{q=1}^{\infty} \sum_{s=1}^{\infty} \frac{(-1)^{q+s-1} q^{s-2} s^{-(q+s)} \Gamma(q+s)}{\Gamma(q) \Gamma(s)} .
$$

According to the relations $B(q, s)=\frac{\Gamma(q) \Gamma(s)}{\Gamma(q+s)}$ and $\alpha \cdot \Gamma(\alpha)=\Gamma(\alpha+1),(\alpha>0)$, the latter expression implies: 
$\int_{0}^{1} x^{x^{x}} d x=\sum_{q=1}^{\infty} \sum_{s=1}^{\infty} \frac{(-1)^{q+s-1} q^{s-2} s^{-(q+s)}}{B(q, s)}=G(q, s)=0.5731215670$

Similar to the series in (7), this double series also contains two infinite families $G(q, s)$. One family of series rises for each fixed $s$ when $q$ varies from 1 to $\infty$ and the other family for each fixed $q$ when $s$ varies from 1 to $\infty$. Namely, fixing $q=1$, we have "Sophomore's dream" again, and setting $q=s$, the diagonal series $\Delta(s)$

$$
G(s, s)=\Delta(s)=\sum_{s=1}^{\infty} \frac{(-1)^{2 s-1} s^{-(S+2)}}{B(s, s)}=\sum_{s=1}^{\infty} \frac{(-1)^{2 s-1} s^{-s-2} 4^{s} \Gamma\left(s+\frac{1}{2}\right)}{2 \sqrt{\pi} \Gamma(s)} .
$$

One could continue this integration process on the interval $I=[0,1]$ for increasingly higher levels of gradients $G_{k}(x)$, with the help of Maple, and get the results shown in Table 1.

This set of data in Table 1 gives us many insights regarding the natural inner properties of the envelope gradient functions. If we keep solving definite integrals on the uniform distribution, we get remarkable results.

\section{TABLE 1}

\section{EXPLORATORY DATA ANALYSIS OF TYPE I QCG MODELS}

Area of the regions bounded by the graph of $G_{k}(x)$ and the $x$-axis, between 0 and 1 .

\begin{tabular}{|c|c|c|c|}
\hline$k$ & $G_{k}(x)$ & $\int_{0}^{1} G_{k}(x) d x$ & Meanings of each result in the square $I \times I$ (see Figure 1$)$ \\
\hline 1 & $G_{1}(x)=x$ & 0.5000000000 & $\begin{array}{l}\text { The first odd level gradient, diagonal of the squared envelope; } 50 \% \\
\text { of data remains below its curve. }\end{array}$ \\
\hline 2 & $\begin{array}{l}G_{2}(x) \\
=x^{x}\end{array}$ & 0.7834305107 & $\begin{array}{l}\text { The first even level gradient contains approximately } 78.34 \% \text { of data } \\
\text { under its curve. }\end{array}$ \\
\hline 3 & $\begin{array}{l}G_{3}(x) \\
=x^{x^{x}}\end{array}$ & 0.5731215670 & $\begin{array}{l}\text { The second odd level gradient contains approximately } 57.31 \% \text { of } \\
\text { data under its curve and } G_{1}(x) \leq G_{3}(x)\end{array}$ \\
\hline 4 & $\begin{array}{l}G_{4}(x) \\
=x^{x^{x^{x}}}\end{array}$ & 0.7313397799 & Approximately $73.13 \%$ of data is under $G_{4}$ and $G_{2}(x) \geq G_{4}(x)$ \\
\hline 5 & $\begin{array}{l}G_{5}(x) \\
=x^{x^{x^{x}}}\end{array}$ & 0.5975776349 & Approximately $59.76 \%$ of data and $G_{1}(x) \leq G_{3}(x) \leq G_{5}(x)$ \\
\hline 6 & $G_{6}(x)=$ & 0.7106589414 & $\approx 71.07 \%$ of data and $G_{2}(x) \geq G_{4}(x) \geq G_{6}(x)$ \\
\hline 7 & $G_{7}(x)=$ & 0.6088236675 & $\approx 60.88 \%$ of data and $G_{1}(x) \leq G_{3}(x) \leq G_{5}(x) \leq G_{7}(x)$ \\
\hline 8 & $G_{8}(x)=$ & 0.7002371602 & $\approx 70 \%$ of data and $G_{2}(x) \geq G_{4}(x) \geq G_{6}(x) \geq G_{8}(x)$ \\
\hline 9 & $G_{9}(x)=$ & 0.6149950813 & $\approx 61.50 \%$ of data, $G_{1}(x) \leq G_{3}(x) \leq G_{5}(x) \leq G_{7}(x) \leq G_{9}(x)$ \\
\hline 10 & $G_{10}(x)=$ & 0.6941860894 & $\approx 69.42 \%$ of data, $G_{2}(x) \geq G_{4}(x) \geq G_{6}(x) \geq G_{8}(x) \geq G_{10}(x)$ \\
\hline ... & $\ldots \ldots \ldots$ & & \\
\hline 29 & $G_{29}(x)=$ & 0.6280216768 & $\approx 62.80 \%$ of data and $G_{1}(x) \leq G_{3}(x) \leq \ldots \leq G_{27}(x) \leq G_{29}(x)$ \\
\hline 30 & $G_{30}(x)=$ & 0.6803851519 & $\approx 68.04 \%$ of data, $G_{2}(x) \geq G_{4}(x) \geq \ldots \geq G_{28}(x) \geq G_{30}(x)$ \\
\hline
\end{tabular}

We intend to publish these computations very soon (see Endnotes).

Since we are dealing with a sequence $G_{k}(x):[a, b] \subset \mathbb{R}_{+} \rightarrow \mathbb{R}_{+}$of real-variable smooth functions, one can establish the following definition: 


\section{Definition of Type I QCG Models}

We recall the consequences of Definition A. Let $\left\{G_{k}(x), k=1,2,3, \ldots\right\}$ be a sequence of GCF that contains the subsequences $\left\{G_{2 k-1}(x), k=1,2,3, \ldots\right\}$ and $\left\{G_{2 k}(x), k=1,2,3, \ldots\right\}$. We pick a member from each subsequence, for each fixed $k$, and define a set of models:

$\Psi_{k}(x)= \begin{cases}\alpha_{1}+\alpha_{2} G_{2 k-1}(x), & \left(\alpha_{1} \geq 0, \alpha_{2}>0\right) \\ \beta_{1}+\beta_{2} G_{2 k}(x), & \left(\beta_{1}, \beta_{2}>0\right)\end{cases}$

These models involve members chosen one from each subsequence (see Figure $3 \mathrm{a}$ and $3 \mathrm{~b}$ ). But we also could construct models selecting both members either from even levels or odd levels, for distinct purposes of the study. We will return to (10) in the next section.

\section{QCG Type II Models}

Proposition 1. The set of all smooth gradient functions $\left\{G_{k}(x)\right\}$ defined pointwise on the half-real line $\mathbb{R} \mathbb{R}_{+}$ is a linear vector space under scalar multiplication and pointwise addition, given by $\varphi_{i j}(x)=\alpha G_{i}(x)+$ $\beta G_{j}(x)$, where $\alpha, \beta$ are real numbers and $i, j=1,2,3, \ldots$ This vector space is an associative algebra under pointwise multiplication of functions $\Phi_{i j}(x)=G_{i}(x) \times G_{j}(x)$.

Proof

Let $G_{0}=0, G_{1}, G_{2}, \ldots \in C^{\infty}\left(\mathbb{R}_{++}^{2}\right)$ be a collection of gradient functions and let $\alpha_{1}, \alpha_{2}, \alpha_{3}, \ldots \in \mathbb{R}$ be scalars in the real field. We can easily verify that $\left(C^{\infty}\left(\mathbb{R}_{++}^{2}\right),+\right)$ is an abelian group under + , with the identity $G_{0}$. Also, $\left(C^{\infty}\left(\mathbb{R}_{++}^{2}\right),+,\right)$ complies with four additional postulates under scalar multiplication . For the third operation $\times$, for any $i, j$, the following is clearly valid: closure, if $G_{i}, G_{j} \in C^{\infty}(x) \rightarrow G_{i} \times G_{j} \in$ $C^{\infty}(x)$. Bilinearity is obvious and associative with $\left(G_{i} \times G_{j}\right) \times G_{r}=G_{i} \times\left(G_{j} \times G_{r}\right)$; the existence of identity is an optional property; symmetry under interchange is met with $G_{i} \times G_{j}=G_{j} \times G_{i}$; and derivative property is given with $G_{i} \times\left(G_{j} \times G_{r}\right)=\left(G_{i} \times G_{j}\right) \times G_{r}+G_{j} \times\left(G_{1} \times G_{r}\right)$. Hence, $\left(C^{\infty}\left(\mathbb{R}_{++}^{2}\right),+,, \times\right)$ is an associative and commutative algebra of smooth gradient functions. $\triangleleft$

\section{Definition of Type II QCG Models}

Let $\left\{G_{k}(x): k=1,2,3, \ldots\right\}$ be a sequence of smooth gradient functions which are elements of the algebra meeting Proposition 1. We define a family of Type II QCG Models with:

$\Theta_{k}(x)=\left\{\begin{array}{l}\alpha_{1} G_{2 k-1}(x)+\alpha_{2} G_{2 k+1}(x)+\alpha_{1} \alpha_{2} G_{2 k-1}(x) \times G_{2 k+1}(x),\left(\alpha_{1}, \alpha_{2}>0\right) \\ \beta_{1} G_{2 k}(x)+\beta_{2} G_{2 k+2}(x)+\beta_{1} \beta_{2} G_{2 k}(x) \times G_{2 k+2}(x),\left(\beta_{1}, \beta_{2}>0\right)\end{array}\right.$

E.g., for $k=1, \Theta_{1}(x)=2 x+3 x^{x^{x}}+6 x \cdot x^{x^{x}}$ is of odd levels and $\Theta_{1}(x)=3 x^{x}+5 x^{x^{x^{x}}}+15 x^{x} \cdot x^{x^{x^{x}}}$ is of even levels, the alpha-curve and the beta-curve, respectively.

Proposition 2. There is a one-to-one amalgamated correspondence between odd level models and even level models defined in (10), (11), (12), and (13).

Proof

It is easy to exhibit a one-to-one correspondence between these types of sets of models and the set of positive integers. Consider the functions $f(k)=2 k-1$ and $g(k)=2 k$ from $\mathbb{Z}_{+}$onto the set of odd levels $\left\{G_{2 k-1}\right\}$ and the set of even levels $\left\{G_{2 k}\right\}$, respectively. These functions are one-to-one and onto. 


$$
\begin{array}{llll}
\square_{+} & \stackrel{f}{\rightarrow}\left\{G_{2 k-1}\right\} & k: 1,2,3,4, \ldots, & \downarrow \\
\mathrm{g} \square & \square h & 2 k-1: 1,3,5,7, \ldots, & \downarrow \\
& \left\{G_{2 k}\right\} & 2 k: 2,4,6,8, \ldots, & \downarrow
\end{array}
$$

The next proposition gives a special consequence of the general definition of these models.

Proposition 3. If we let $\tau_{2 k}(x)=\beta_{1} G_{2 k}(x)+\beta_{2} G_{2 k+2}(x)+\beta_{1} \beta_{2} G_{2 k}(x) \times G_{2 k+2}(x)$ be a set of even level of $\beta$-curve $Q C G$ Models, then

$\Theta_{k}(x)=\left\{\begin{array}{l}\alpha_{1} G_{2 k-1}(x)+\alpha_{2} G_{2 k+1}(x)+\alpha_{1} \alpha_{2} G_{2 k-1}(x) \times G_{2 k+1}(x),\left(\alpha_{1}, \alpha_{2}>0\right) \\ \left(\beta_{1}+\beta_{2}\right)^{2}-\tau_{2 k}(x),\left(\beta_{1}, \beta_{2}>0\right)\end{array}\right.$

The proof is simply a reflected transformation of a closed envelope into an open envelope, as we show in the next section. To have a concrete geometrical view, see figures $4 \mathrm{a}$ and $4 \mathrm{~b}$.

\section{Type III QCG Models}

In this section, additionally to the previous elements of the type I and II models, we use definitions B, $\mathrm{C}$, and D. Our purpose is to deform the algebra $C^{\infty}\left(\mathbb{R}_{++}^{2}\right)$ stated in Proposition 1 to $C_{*}^{\infty}\left(\mathbb{R}_{++}^{2}\right)$ algebra.

We start with the pointwise commutative product $\times$ on $C^{\infty}\left(\mathbb{R}_{++}^{2}\right)$ and we want to define a family of products $*_{\hbar}$ depending on a parameterh such that when $\hbar=0, *_{0}=\times$. I.e., we ask for the expression $*_{\hbar}$ to be a formal power series, so we do not need to worry about convergence. However, then $G_{i} \times G j$ is no longer in $C^{\infty}\left(\mathbb{R}_{++}^{2}\right)$, but in $C_{*}^{\infty}\left(\mathbb{R}_{++}^{2}\right) \llbracket \hbar \rrbracket$. It seems confusing, since we expect a product to involve two elements in the algebra $C^{\infty}\left(\mathbb{R}_{++}^{2}\right)$ and give an element in the same algebra. To solve this, one can extend the product to the $C_{*}^{\infty}\left(\mathbb{R}_{++}^{2}\right) \llbracket \hbar \rrbracket$ algebra by $\mathbb{R} \llbracket \hbar \rrbracket$ - linearity, by Definition $D$. Hereinafter, $\hbar$ is no longer a parameter, but a formal variable. We are thus ready to define the models.

\section{Definition of Type III QCG Models}

Let $\left\langle C^{\infty}\left(\mathbb{R}_{++}^{2}\right),+, \times\right\rangle$ be a commutative and associative algebra or simply $C^{\infty}\left(\mathbb{R}_{++}^{2}\right)$, established in Proposition 1. Let $G_{i}, G_{j} \in C^{\infty}\left(\mathbb{R}_{++}^{2}\right)$ be smooth gradient real-valued functions of level $i$ and $j$, respectively. We define a family of Type III QCG Models, denoted by $G_{i} *_{\hbar} G j=G_{i} * G j=\Upsilon_{k}$, as

$\Upsilon_{k}=\left\{\begin{array}{l}\sum_{n=0}^{\infty} \frac{1}{n !} \frac{\hbar^{n}}{2^{n}} B_{n}\left(G_{2 k-1}, G_{2 k+1}\right), \text { for odd level of } G_{i}^{` s} \\ -\sum_{n=0}^{\infty} \frac{1}{n !} \frac{\hbar^{n}}{2^{n}} B_{n}\left(G_{2 k}, G_{2 k+2}\right), \text { for even level of } G_{i}^{\text {s }}\end{array}\right.$

where $B_{n}$ is either a Poisson bivector or a bidifferential operator based on definition D. In particular, $B_{0}\left(G_{i}, G_{j}\right)=G_{i} G_{j}$ is the ordinary commutative product in the classical limit of $\hbar \rightarrow 0$ and $B_{1}\left(G_{i}, G_{j}\right)=$ $\left\{G_{i}, G_{j}\right\}=\frac{\partial G_{i}}{\partial q} \frac{\partial G_{j}}{\partial p}-\frac{\partial G_{i}}{\partial p} \frac{\partial G_{j}}{\partial q}$ is the Poisson bracket. More generally, $B_{n}\left(G_{i}, G_{j}\right)=$ $\sum_{r=0}^{n}(-1)^{r}\left(\begin{array}{c}n \\ r\end{array}\right)\left(\frac{\partial^{r}}{\partial p^{r}} \frac{\partial^{n-r}}{\partial q^{n-r}} G_{i}\right) \times\left(\frac{\partial^{n-r}}{\partial p^{n-r}} \frac{\partial^{r}}{\partial q^{r}} G_{j}\right)$. Here, $\left(q_{s}, p_{s}\right)$ are canonical coordinates on the phase space $\mathbb{R}_{++}^{2}$. For an illustration, see the next section.

\section{SUPPORTING METHODOLOGY AND APPLICATIONS}

Here we present the simplest concrete cases of these infinite three types of QCG Models to show how they help us in the exploratory analysis of financial markets. A variety of well-known topics in business are focused on this new point of view. The frame of our work $\mathbb{R}_{++}^{2} \subset \mathbb{R}^{d}$ is also the simplest two-dimensional Poisson manifold, phase space. We present one case for each type of QCG Model, starting with Type I. 
Let us take equation (10) and make some changes to its general structure. The reader may already suspect what famous law of economics these equations look like:

$\Psi_{k}(x)=\left\{\begin{array}{l}-\alpha_{1}+\alpha_{2} G_{2 k-1}(x),\left(\alpha_{1} \geq 0, \alpha_{2}>0\right) \\ \beta_{1}-\beta_{2} G_{2 k}(x), \quad\left(\beta_{1}, \beta_{2}>0\right)\end{array}\right.$

Let's include the condition of equilibrium into these models: the point where the $\alpha$-curves and the $\beta$ curves meet. That is, $-\alpha_{1}+\alpha_{2} G_{2 k-1}(x)=\Psi_{k}(x)=\beta_{1}-\beta_{2} G_{2 k}(x)$. From these three equations, we establish the law of supply and demand and find the equilibrium solutions of endogenous variables, as $x$ denotes the price of a good in the market. Concretely, we proceed to start with $k=1, k=2$, and so on, as follows:

$$
\begin{aligned}
& \Psi_{1}(x)\left\{\begin{array}{l}
-\alpha_{1}+\alpha_{2} x, x \geq 0 \\
\beta_{1}-\beta_{2} x^{x}, x \geq 0
\end{array} \text { and }-\alpha_{1}+\alpha_{2} x=\beta_{1}-\beta_{2} x^{x}\right. \\
& \Psi_{2}(x)\left\{\begin{array}{l}
-\alpha_{1}+\alpha_{2} x^{x^{x}}, x \geq 0 \\
\beta_{1}-\beta_{2} x^{x^{x^{x}}}, x \geq 0
\end{array} \text { and }-\alpha_{1}+\alpha_{2} x^{x^{x}}=\beta_{1}-\beta_{2} x^{x^{x^{x}}}\right.
\end{aligned}
$$

where the parameters $\alpha$ and $\beta$ are not necessarily the same and may be chosen arbitrarily considering a specific interaction in the markets. At this point, we have a whole range of supply and demand models to analyze the equilibrium of markets in economics, the equilibrium of national income, and other financial variables. Also, one can use these models to study the behavior of a single good in a single market, many goods in a single market, as well as many goods in many markets, simultaneously. Furthermore, the best aspect of these models is that they all comply with the rules of standard and stochastic calculus. See the natural behavior of shapes of (15), which give rise to all well-known areas studied in economics, for some specific parameter values. We solve the equilibrium equation $\Psi_{s}(x)=\Psi_{d}(x)$ and we find the point $\mathrm{E}(2.6,10)$ (see Figure 3a). Notice that, in order to keep the meaning of a function, we have plotted without switching the axis around.

\section{FIGURE 3A MARKET STATIC EQUILIBRIUM TYPE 1 MODEL FOR k=1}

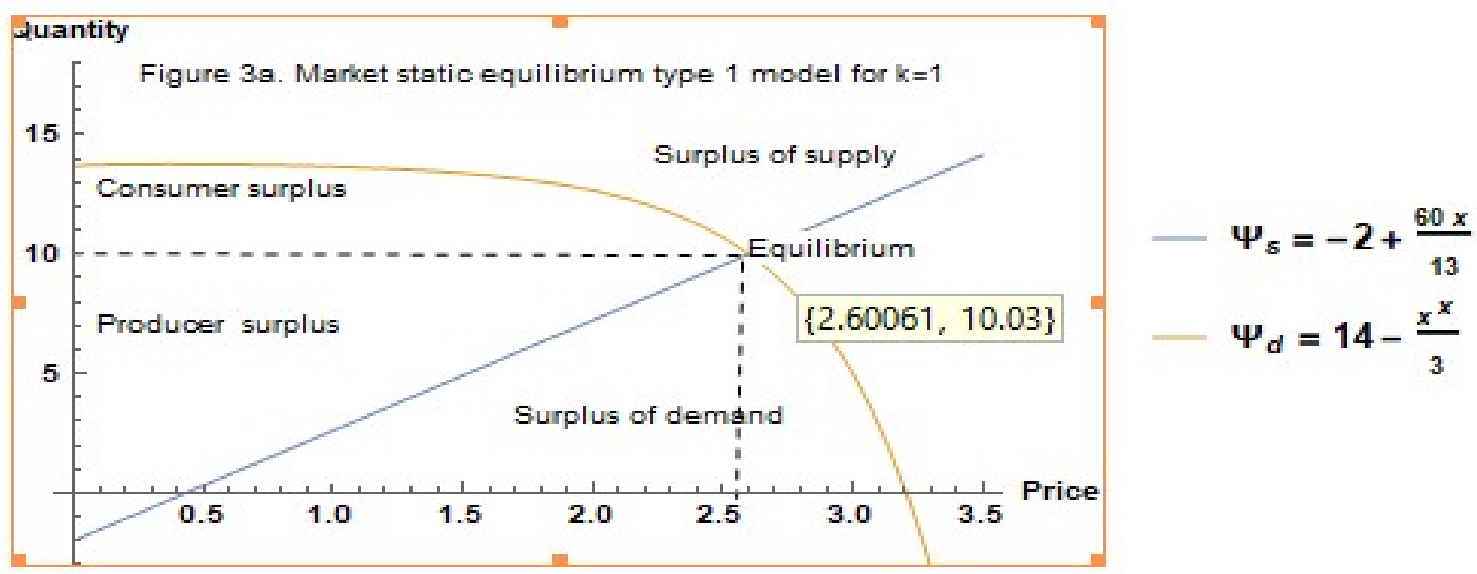

Similarly, we proceed to model (16). One can arbitrarily choose some parameter values selecting data from the markets and solving the equations. In this case, we have: 


$$
\Psi_{2}(x)\left\{\begin{array}{l}
-0.5+2 x^{x^{x}}, x \geq 0 \\
14-x^{x^{x^{x}}}, x \geq 0
\end{array} \text { and }-0.5+2 x^{x^{x}}=14-x^{x^{x^{x}}}\right.
$$

In this case, we have solved the equilibrium equation $-0.5+2 x^{x^{x}}=14-x^{x^{x^{x}}}$ by applying the numerical approximation method, obtaining the equilibrium point $\mathrm{E}(1.7,7)$. The quantity has to be an integer number. It means that if the price of a good or service is $\$ 1.7$, then the quantity of a good or service supplied by producers is 7 units of goods or services and it is equal to the quantity demanded by consumers. We illustrate these shapes of the curves and show the results in Figure $3 \mathrm{~b}$.

\section{Corollary 1}

Take the statement of Theorem H and Proposition 2. In equation (14), (17), and (21), for each $k=$ $1,2,3, \ldots$, there is a 1 to 1 correspondence between the Law of Supply ( $\alpha$-curves) and the Law of Demand ( $\beta$-curves). The proof is the same as proposition $2 . \triangleleft$

FIGURE 3B

MARKET STATIC EQUILIBRIUM TYPE 1 MODEL FOR k=2

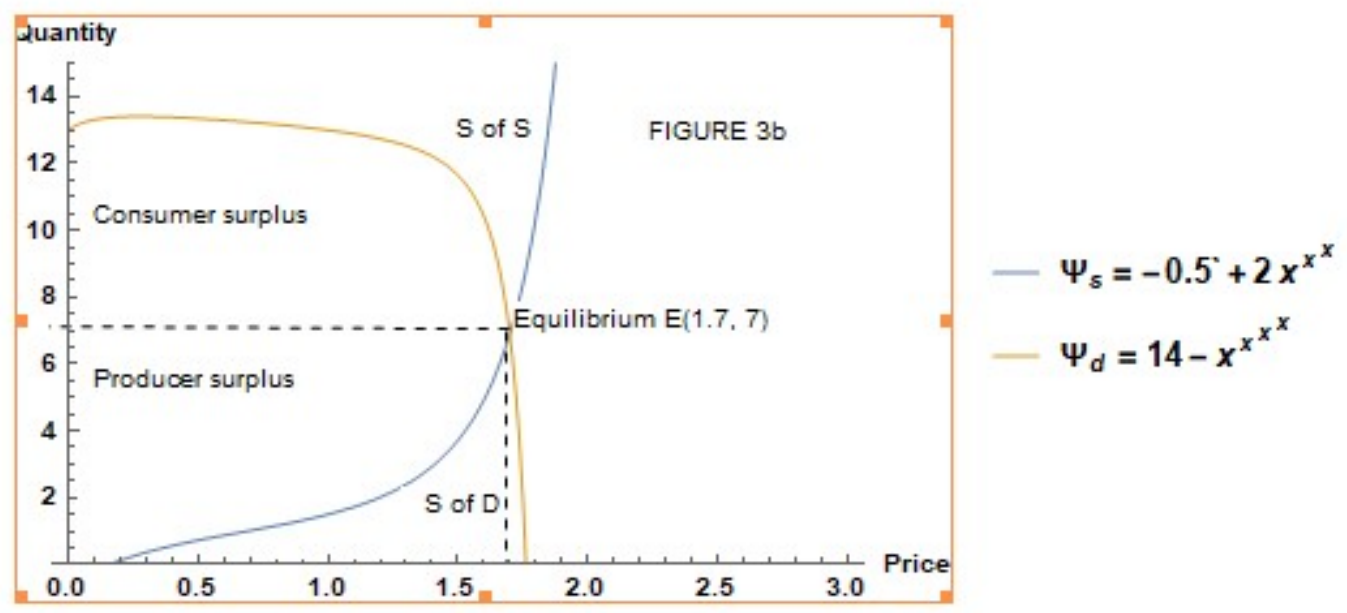

Next, we present a specific application of Type II GCF models. Here, we use Definition A, the definition in (11) and we take Proposition 3 as a special case. Let's take the simplest case $k=1$ and $k=2$ :

$$
\begin{aligned}
& \Theta_{1}(x)=\left\{\begin{array}{l}
\alpha_{1} G_{1}(x)+\alpha_{2} G_{3}(x)+\alpha_{1} \alpha_{2} G_{1}(x) G_{3}(x) \\
\left(\beta_{1}+\beta_{2}\right)^{2}-\beta_{1} G_{2}(x)-\beta_{2} G_{4}(x)-\beta_{1} \beta_{2} G_{2}(x) G_{4}(x)
\end{array}\right. \\
& \Theta_{2}(x)=\left\{\begin{array}{l}
\alpha_{1} G_{3}(x)+\alpha_{2} G_{5}(x)+\alpha_{1} \alpha_{2} G_{3}(x) G_{5}(x) \\
\left(\beta_{1}+\beta_{2}\right)^{2}-\beta_{1} G_{4}(x)-\beta_{2} G_{6}(x)-\beta_{1} \beta_{2} G_{4}(x) G_{6}(x)
\end{array}\right.
\end{aligned}
$$

We may study a wide variety of market dynamics through the application of these models choosing some $\alpha$ and $\beta$ parameter values, according to the researcher's purpose. To keep our goal, we focus on the law of supply and demand for $\alpha_{1}=2, \alpha_{2}=\beta_{1}=3, \beta_{2}=5$ :

$\Theta_{1}(x)=\left\{\begin{array}{l}2 x+3 x^{x^{x}}+6 x \cdot x^{x^{x}} \\ 64-3 x^{x}-5 x^{x^{x^{x}}}-15 x^{x} \cdot x^{x^{x^{x}}}\end{array}\right.$ 


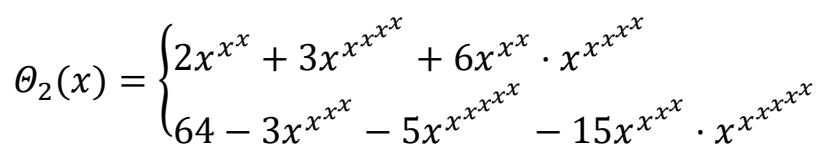

Plotting their graphs, we amazingly see again all traditional areas on which the laws of economics and finance interact. But, in this case, since we deal with GCFs, elements of an associative algebra $C\left(\mathbb{R}_{++}^{2}\right)$, the analysis of economics is the classical dynamical field. One should consider $x$ the price of a good and $x(t)$ the price depending on time, and we can study the specific trajectories of instantaneous market changes over time. Again, the standard differential and integral calculus is the right tool. For a vision of the picture underlying this analytical approach, a mapped Poisson manifold $\mathbb{R}_{++}^{2}$, see Figure $4 \mathrm{a}-4 \mathrm{~b}$.

FIGURE 4A

\section{DYNAMIC EQUILIBRIUM TYPE 2 MODEL FOR $\mathrm{k}=1$}

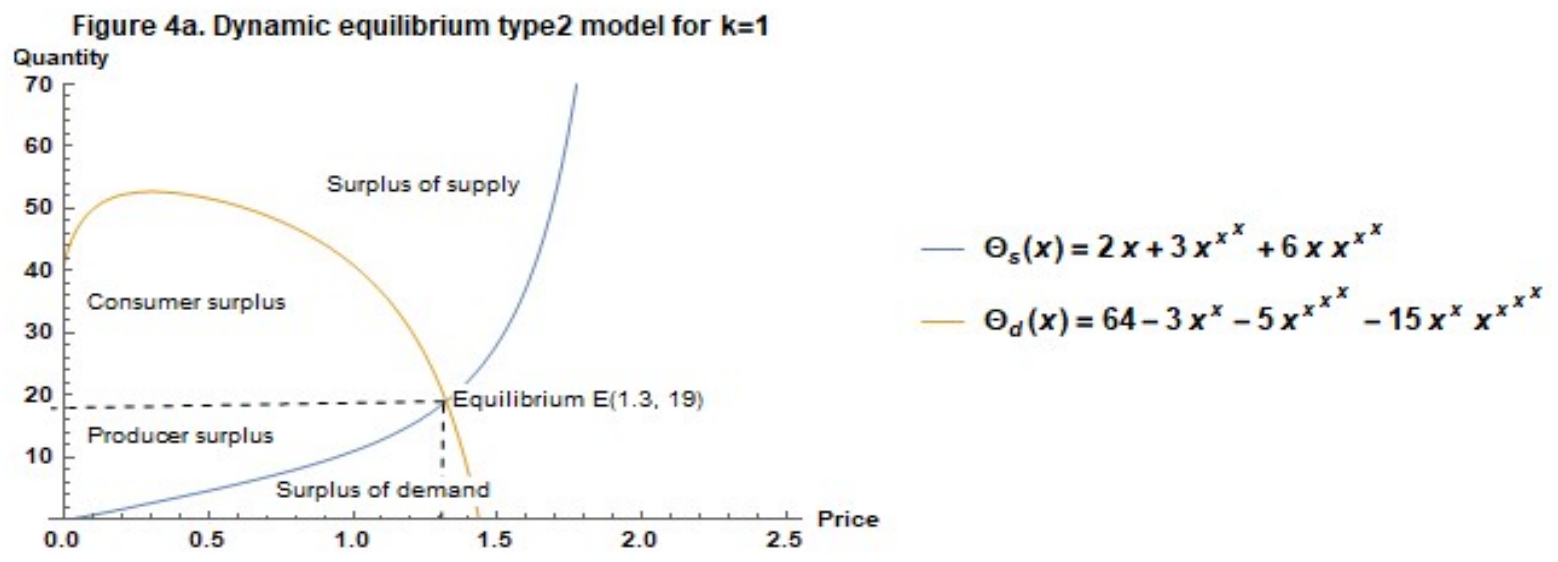

To find the equilibrium point $E(1.33,18$ or 19$)$, we have solved the equation $\Theta_{s}(x)=\Theta_{d}(x)$ by applying the numerical approximation method with Mathematica and verifying it with Maple. It means that if the price of a good or service is $\$ 1.3281$, then producers supply 18 or 19 units of goods or services and it is equal to the quantity demanded by consumers. We illustrate these shapes of the curves and show the results in Figure 4a.

FIGURE 4B

DYNAMIC EQUILIBRIUM TYPE 2 MODEL FOR k=2

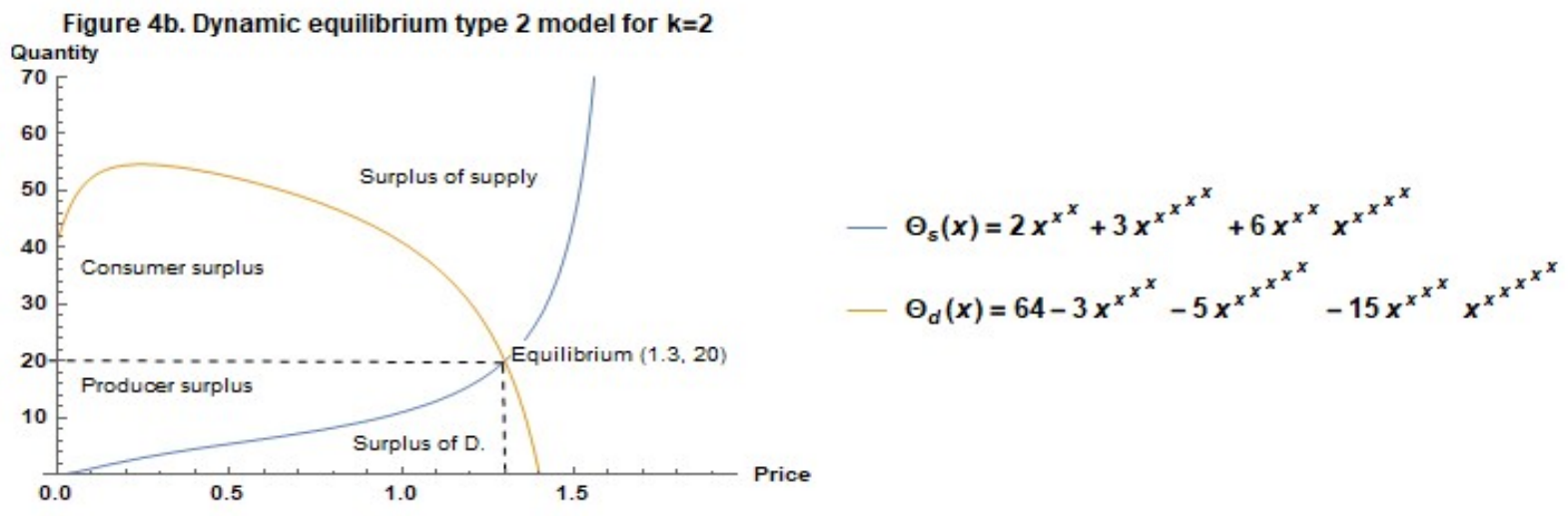


Once again, the equilibrium point $E(1.3,20)$ is determined solving the equation $\Theta_{S}(x)=\Theta_{d}(x)$ by using the numerical approximation method with Mathematica and Maple (see illustration in Figure 4b).

Next, we explain a specific case of Type III GCF Models. But now we apply these models in the quantum field. Punctually, we want to deform the associative algebra defined on a Poisson manifold $\mathbb{R}_{++}^{2}$, the phase space, whose observable of the markets are the smooth functions. From its definition (13), they can be written as:

$$
\Upsilon_{k}(x)=\left\{\begin{array}{lc}
B_{0}\left(G_{2 k-1}, G_{2 k+1}\right)+\hbar B_{1}\left(G_{2 k-1}, G_{2 k+1}\right)+\vartheta\left(\hbar^{2}\right) & \alpha \text {-curve } \\
-B_{0}\left(G_{2 k}, G_{2 k+2}\right)+\hbar B_{1}\left(G_{2 k}, G_{2 k+2}\right)+\vartheta\left(\hbar^{2}\right) & \beta \text {-curve }
\end{array}\right.
$$

The term $\vartheta\left(\hbar^{2}\right)$ is a truncation of $\Upsilon_{k}(x)$ that contains the rest of the terms of the formal power series. We consider Dirac's original scheme (Dirac P.A.M. 1930) of deformation quantization and (13) can only be taken up to its linear term, while (21) will be a good approximation to the complete deformation. In particular:

$\Upsilon_{k}(x)=\left\{\begin{array}{l}G_{2 k-1} G_{2 k+1}+\hbar\left(\frac{\partial G_{2 k-1}}{\partial q} \frac{\partial G_{2 k+1}}{\partial p}-\frac{\partial G_{2 k-1}}{\partial p} \frac{\partial G_{2 k+1}}{\partial q}\right) \\ -G_{2 k} G_{2 k+2}+\hbar\left(\frac{\partial G_{2 k}}{\partial q} \frac{\partial G_{2 k+2}}{\partial p}-\frac{\partial G_{2 k}}{\partial p} \frac{\partial G_{2 k+2}}{\partial q}\right)\end{array}\right.$

The simplest case for $k=1$ :

$$
\Upsilon_{1}(x)=\left\{\begin{array}{l}
G_{1} G_{3}+\hbar\left(\frac{\partial G_{1}}{\partial q} \frac{\partial G_{3}}{\partial p}-\frac{\partial G_{1}}{\partial p} \frac{\partial G_{3}}{\partial q}\right) \\
-G_{2} G_{4}+\hbar\left(\frac{\partial G_{2}}{\partial q} \frac{\partial G_{4}}{\partial p}-\frac{\partial G_{2}}{\partial p} \frac{\partial G_{4}}{\partial q}\right)
\end{array}=\left\{\begin{array}{l}
x \cdot x^{x^{x}}+\hbar\left(\frac{\partial x}{\partial q} \frac{\partial x^{x^{x}}}{\partial p}-\frac{\partial x}{\partial p} \frac{\partial x^{x^{x}}}{\partial q}\right) \\
-x^{x} \cdot x^{x^{x^{x}}}+\hbar\left(\frac{\partial x^{x}}{\partial q} \frac{\partial x^{x^{x^{x}}}}{\partial p}-\frac{\partial x^{x}}{\partial p} \frac{\partial x^{x^{x^{x}}}}{\partial q}\right)
\end{array}\right.\right.
$$

Here, $q$ and $p$ are canonical coordinates, presented above. We remark that $G_{i}(x)=G_{i}(q, p ; x)$. E.g., we should name $q=$ position operator (price at a specific date) and $p=$ impulsion operator (time at which a price changes). The formal expansion (deformation) depends on the variable $\hbar$ and it is any positive real number. One can assign many real values and analyze the conduct and properties of $\Upsilon_{1}(x)$. The exceptional case occurs when $\hbar=0$. We return to classical mechanics, classical economics. It is the correspondence principle of QM. We plot this case with a little modification, see Figure 5, and, again, we find the domain of interaction of the law of supply and demand, the $\alpha$-curve and the $\beta$-curve, respectively.

\section{FIGURE 5}

\section{QUANTUM EQUILIBRIUM TYPE 3 MODEL FOR $\mathrm{k}=1$ AND $\mathrm{h}=0$}

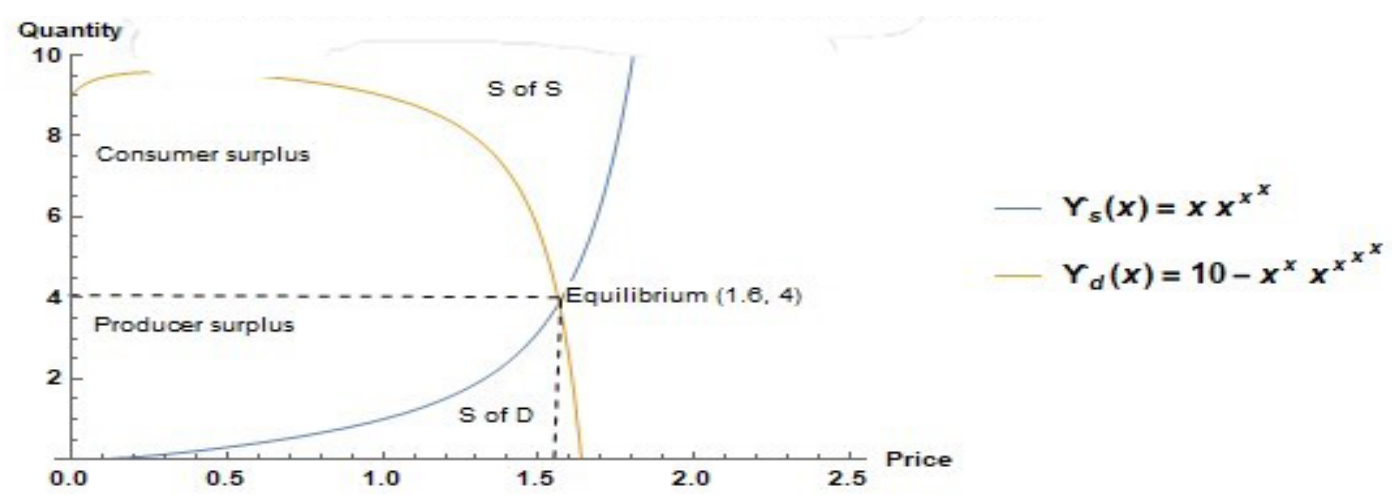




\section{The $\alpha \boldsymbol{\beta}$ Conjecture}

Let $C^{\infty}(M)$ be an associative algebra of smooth functions on a Poisson manifold $M$ The $(\alpha-$ curve equations $)-(\beta$-curve equations $)=0$ is the quantum equilibrium of the law of supply and demand in the markets. This could also be valid for other natural laws.

\section{CONCLUSIONS}

In this paper, we have built and described a few types of quantum continuous gradient models (QCGM) to study and analyze the interaction of economic variables from two points of view: the classical mechanics (type I and type II models) and quantum mechanics (type III models). In particular, we have focused on the variables that describe the behavior of goods and services in financial markets. This infinite set of models has been built on a Poisson manifold $\mathbb{R}_{++}^{2}$ whose basic ingredient is a sequence of continuous gradient functions $\left\{G_{k}(x): k=1,2,3, \ldots\right\}$ bifurcated into two subsequences $\left\{G_{2 k-1}(x): k=1,2,3, \ldots\right\}$ and $\left\{G_{2 k}(x): k=1,2,3, \ldots\right\}$, Lemma 1 and Theorem H. These QCG Models have been classified into three families: Type I models, Type II models, and Type III models. In the study process, it has been observed that for each level of order $k$ in every three types of models, $\Psi_{k}, \Theta_{k}$, and $\Upsilon_{k}$, there is an injective correspondence between an element of odd order ( $\alpha$-curve) with another element of even order ( $\beta$-curve) and they meet at the equilibrium point, demonstrated in Proposition 2. A special case focused on the law of supply and demand shown in corollary 1 . From the point of view of economics, models of type A are static classical models that show us an underlying framework of work traditional in economics and finance over which any economic variable can be studied. For example, the case of the supply and demand curves shows us different regions in figures $3 \mathrm{a}$ and $3 \mathrm{~b}$ that have economic, financial, and probabilistic statistical significance, shown in Table 1. A more exhaustive treatment of the study of this type of model would lead to the analysis of optimization of these regions, through this family of models, but it was not the goal of this study. However, another study on that topic will be published soon.

Type II models are dynamic models that involve, in their structure, the time variable $t$ and $X(t)$ as trajectories and because the original elements $G_{k}(x)$ are now elements of an associative algebraic structure, Proposition 1. These Type II models are more general than Type I models.

Finally, the Type III models are quantum dynamic models obtained by deformation of the underlying frame of Type II models, the associative algebra $C^{\infty}\left(\mathbb{R}_{++}^{2}\right)$ of $\left\{G_{k}(x)\right\}$. This could be a case of connection between the economic models of classical mechanics and the models of quantum mechanics. We have explained very little about the deep and complex contents of this Type III model, focusing on the generalized supply and demand law. Further results will be presented in the next part of this paper.

\section{RECOMMENDATIONS}

It is well known from econophysics that classical economics and quantum economics are two worlds in which variables such as price, supply, demand, and whatever is the variable one wants to study interact. It is also well known that phenomena in the quantum description are more detailed than the classical one. Thus, we recommend to keep researching in this sense because it should be the most robust and complete horizon of economics and finance.

Furthermore, we dare to conjecture that Type III models are a generalization of the previous ones, but their development is very difficult even though we use mathematical software. For example, if we analyze the behavior of the supply and demand curves in the three types of models, we see that the Type II and Type III models are more general in the sense that they show the demand curve with a positive (describing the diamond and Veblen goods demand) and negative slope, therefore zero slopes, and possible infinite slope as the price of a good or service changes over time. The proposal of these models is at the discretion of the authors, and we believe that this is possibly a new path where modern economy is studied within a quantum context. We have represented models mathematically using quantum deformation theory. 
However, there is still a whole universe to discover and develop in this open field of the quantum theory of equilibrium, considering the $\alpha \beta$-conjecture.

Finally, we suggest looking for other economic and financial meanings of these definite integrals. In particular the one presented in Table 1, page 9, from equation (2).

Letting $b=e^{-1}$ in (2), we solve the integral:

$$
\begin{aligned}
& \int_{0}^{\frac{1}{e}} x^{x^{x}} d x=\sum_{q=0}^{\infty} \sum_{p=0}^{\infty} \frac{q^{p}}{p ! q !} \int_{0}^{\frac{1}{e}}\left(x^{p} \ln ^{p+q} x\right) d x \\
& =\sum_{q=0}^{\infty} \sum_{p=0}^{\infty} \Phi(p, q)\left(\begin{array}{l}
(1+p)^{-\left(\frac{p+q+2}{2}\right)} e^{-\left(\frac{p+1}{2}\right)} \text { Whitta ker } M\left(\frac{p+q}{2}, \frac{p+q+1}{2}, p+1\right) \Gamma(-p-q) \sin (\pi p) \cos (\pi q)+ \\
(1+p)^{-\left(\frac{p+q+2}{2}\right)} e^{-\left(\frac{p+1}{2}\right)} \text { Whitta ker } M\left(\frac{p+q}{2}, \frac{p+q+1}{2}, p+1\right) \Gamma(-p-q) \cos (\pi p) \sin (\pi q)+ \\
(1+p)^{-(p+q+1)} \pi p+(1+p)^{-(p+q+1)} \pi q+(1+p)^{-(p+q+1)} \pi .
\end{array}\right)
\end{aligned}
$$

where $\Phi(p, q)=\frac{(-1)^{p+q+1} q^{p}}{p ! q !(p+q+1) \Gamma(-p-q) \sin (\pi p+\pi q)}$.

Rewriting and simplifying, we obtain:

$$
=\sum_{q=0}^{\infty} \sum_{p=0}^{\infty} \Phi(p, q)\left(\begin{array}{l}
(1+p)^{-\left(\frac{p+q+2}{2}\right)} e^{-\left(\frac{1+p}{2}\right)} \text { Whitta ker } M\left(\frac{p+q}{2}, \frac{p+q+1}{2}, p+1\right) \Gamma(-p-q) \sin (\pi p+\pi q) \\
+(1+p)^{-(p+q+1)} \pi(p+q+1)
\end{array}\right)
$$

Now, letting $a=\frac{p}{2}+\frac{q}{2}, c=\frac{p}{2}+\frac{q}{2}+\frac{1}{2}$, and $z=1+p$, we get:

$$
\text { Whitta ker } M\left(\frac{p}{2}+\frac{q}{2}, \frac{p}{2}+\frac{q}{2}+\frac{1}{2}, p+1\right)=e^{-\left(\frac{p+1}{2}\right)}(1+p)^{\frac{p+q+2}{2}} \sum_{r=0}^{\infty} \frac{\Gamma(p+q+2)(1+p)^{r}}{\Gamma(p+q+r+2)} \text {. }
$$

Next, we replace the result in the latter expression and multiply by $\Phi(p, q)$. Finally, simplifying it further, we get the following series:

$$
=\sum_{q=0}^{\infty} \sum_{p=0}^{\infty} \frac{(-1)^{p+q+1} q^{p}}{p ! q !}\left(e^{-(p+1)} \Gamma(p+q+1) \sum_{r=0}^{\infty} \frac{(1+p)^{r}}{\Gamma(p+q+r+2)}+\frac{\pi(1+p)^{-(p+q+1)}}{\Gamma(-p-q) \sin (\pi p+\pi q)}\right)
$$

But,

$\sum_{r=0}^{\infty} \frac{(1+p)^{r}}{\Gamma(p+q+r+2)}=\frac{e^{1+p}(1+p)^{-(p+q+1)}(-\Gamma(p+q+1,1+p)+\Gamma(p+q+1))}{\Gamma(p+q+1)}$

and

$\Gamma(p+q+1) \Gamma(-p-q)=\frac{-\pi}{\sin (\pi p+\pi q)}$

Hence, replacing (23) and (24) in the last expression, one finds that the integral of the gradient function $G_{3}(x)$, over the interval $(1,1 / e) \subset[0,1]$ is the double series: 


$$
\sum_{q=0}^{\infty} \sum_{p=0}^{\infty} \frac{(-1)^{p+q+2} q^{p}(1+p)^{-(p+q+1)} \Gamma(p+q+1,1+p)}{p ! q !}
$$

So, we finally get to:

$$
\int_{0}^{\frac{1}{e}} x^{x^{x}} d x==\sum_{q=0}^{\infty} \sum_{p=0}^{\infty} \frac{(-1)^{p+q} q^{p}(1+p)^{-(p+q+1)} \Gamma(p+q+1,1+p)}{p ! q !} \triangleleft
$$

\section{REFERENCES}

Bayen, F., Flato, M., Fronsdal, C., Lichnerowiccz, A., \& Sternheimer, D. (1978). Deformation theory and quantization I-II. Ann. Phys., 111, 61-110, 111-151.

Berezin, F.A. (1975). General Concept of Quantization. Commun. Math. Phys., 40, 153-174. Springer Verlag.

Brown, R.F. (1988). Fixed Point Theory and Its Applications. American Mathematical Society. ISBN: 08218-5080-6

De Wilde, M., \& Lecomte, P. (1998). Formal deformations of the Poisson Lie algebra of symplectic manifold and star-products. In M. Hazewinkel \& M. Gerstenhaber (Eds.), Existence, equivalence, derivations, Deformation Theory of Algebras and Structures and Applications (Dordrecht) (pp. 897-960). Kluwer Acad. Pub.

Dirac, P.A.M. (1930). The Principles of Quantum Mechanics. Clarendon Press, Oxford.

Elliot, R.J., \& Ekkehard Kopp, P. (2005). Mathematics of Financial Markets (Second edition). Springer Science + Business. New York.

Haven, E., \& Khrennikov, A. (2017). The Palgrave Handbook of Quantum Models in Social Science: Applications and Grand Challenges. Macmillan Publisher Ltd. London.

Heisenberg, W. (1930). The physical principles of the quantum theory (K. Eckhart \& F. C. Hoyt Trans.). New York: Dover (rpt. 1949).

Huarca, O.J.M. (2017). The (M,B,Q) Financial Models. Journal of Accounting and Finance, 17(1). ISSN: 2158-3625

Jaeger, G., Khrennikov, A., Schlosshauer, M., \& Weihs, G. (Eds.). (2011). Advances in Quantum Theory. American Institute of Physics Conference Proceedings (Vol. 1327). Melville, NY: American Institute of Physics.

Knuth, D.E. (1976). Mathematics and Computer Science: Coping with Finiteness. Advances in our Ability to Compute and Bringing us Substantially Closer to Ultimate Limitations. Science, 194, $1235-1242$.

Orrell, D. (2019, September 21). Introduction to the mathematics of quantum economics. Retrieved from http://www.postpythagorean.com/quantumeconomicsmath.pdf

Plotnitsky, A. (2016). The Principles of Quantum Theory, From Planck's Quanta to the Higgs Boson. Springer, p.68.

Rippon, P.J. (1983). Infinite Exponentials. Math. Gaz., 67, 189-196.

Ross, S.A., Westerfield, R.W., \& Jaffe, J.F. Finanzas Corporativas. (2000). McGraw Hill Interamericana Editores, S.A. de C.V. Quinta Edición. 